\title{
Ongoing outbreak of West Nile virus infection in humans, Greece, July to August 2011
}

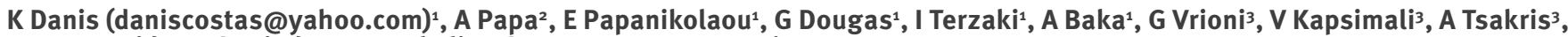

A Kansouzidou ${ }^{4}$, S Tsiodras ${ }^{1}$, N Vakalis ${ }^{5}$, S Bonovas ${ }^{1}$, J Kremastinou ${ }^{1,5}$

1. Hellenic Centre for Disease Control and Prevention, Athens, Greece

2. Department of Microbiology, Aristotle University of Thessaloniki, Thessaloniki, Greece

3. Department of Microbiology, National and Kapodistrian University of Athens, Athens, Greece

4. Department of Microbiology, Infectious Disease Hospital, Thessaloniki, Greece

5. National School of Public Health, Athens, Greece

Citation style for this article:

Danis K, Papa A, Papanikolaou E, Dougas G, Terzaki I, Baka A, Vrioni G, Kapsimali V, Tsakris A, Kansouzidou A, Tsiodras S, Vakalis N, Bonovas S, Kremastinou J.

Ongoing outbreak of West Nile virus infection in humans, Greece, July to August 2011.

Euro Surveill. 2011;16(34):pii=19951. Available online: http://www.eurosurveillance.org/ViewArticle.aspx?Articleld=19951

Article published on 25 August 2011

Between 16 July and 21 August 2011, 31 cases of West Nile neuroinvasive disease were reported from four regions in Greece. Of these, 17 occurred in districts that had not been affected in 2010. The reoccurrence of human cases in two consecutive years (following the large 2010 outbreak) and the spread of the virus in new areas suggest that West Nile virus is established in Greece, and its transmission may continue to occur in the future.

Since July 2011, an outbreak of West Nile virus (WNV) infection has been ongoing in regions in Greece that had already been affected in 2010, and in regions that had never reported human cases before. Here we present information on the extent of the ongoing outbreak and describe the geographical and temporal distribution of West Nile neuroinvasive disease (WNND) cases.

During 2010, Greece experienced the second largest outbreak of WNV infections in Europe since the one that had occurred in Romania in 1996 [1-3]. Overall, 262 cases of WNV infection in humans were notified mainly in northern Greece. In the Central Macedonia region all seven districts had reported cases and in the adjacent Thessalia region one of the four districts was affected. Among reported cases, 197 presented with WNND and 33 of these died, indicating a case fatality rate of $17 \%$ among WNND cases [1]. WNV lineage 2 sequences were obtained from three pools of Culex mosquitoes (strain Nea Santa-Greece-2010), from two blood donors and from wild birds [4-6]. This was the first time that WNV infection was documented in humans in Greece, although serosurveys in the early 1960's, 1980's and 2007 identified WNV antibodies in approximately 1\% of the human population, suggesting that WNV, or a related flavivirus, was circulating in Greece [7-9].

\section{Surveillance methods}

As part of the general surveillance system, physicians in Greece are asked to notify the Hellenic Centre for Disease Control and Prevention (HCDCP) of all cases of WNV infection, using a slightly modified 2008 European Union (EU) case definition (the same as the one used in 2010) $[2,10]$. A confirmed case is defined as a person meeting any of the following clinical criteria: encephalitis, meningitis, fever without specific diagnosis and at least one of the four laboratory criteria: (i) isolation of WNV from blood or cerebrospinal fluid (CSF), (ii) detection of WNV nucleic acid in blood or CSF, (iii) WNV-specific antibody response (IgM) in CSF, and (iv) WNV IgM high titre, and detection of WNV IgG, and confirmation by neutralisation. A case is considered probable if the patient meets the above clinical criteria and a WNV-specific antibody response is demonstrated in his or her serum sample.

A standardised reporting form is used to collect information regarding the demographic characteristics, clinical manifestations, underlying chronic medical conditions, potential risk factors and laboratory results of the reported cases. Regular telephone inquiries to hospitals in the affected areas are conducted for case finding, follow-up and data validation. In addition, indepth telephone interviews are conducted using a semistructured questionnaire to obtain a detailed exposure history of all cases. Cases reported as encephalitis (including meningoencephalitis), meningitis, or acute flaccid paralysis, are classified as WNND cases. All other cases are considered non-neuroinvasive.

\section{Laboratory methods}

Serum and CSF specimens were tested for the presence of WNV-specific IgM and IgG antibodies using commercial ELISA kits (WNV IgM capture DxSelect and WNV IgG DxSelect, Focus Diagnostics Inc, Cypress, CA, USA). WNV positive specimens were also tested for the presence of other flaviviruses: tick-borne encephalitis virus (TBEV) and dengue virus (DENV).

Data analysis

Incidence rates were calculated using as denominator the $\mathbf{2 0 0 7}$ mid-year population estimates of the Hellenic 
Statistical Authority (HSA) [11]. Comparison of categorical variables was assessed using the chi-squared test. Risk ratios (RR) were calculated to compare incidence rates. The analysis was carried out using STATA version 10 software (Stata Corporation LP, Texas, USA). Data were mapped using the GNU R software (www. gnu.org/s/r/).

\section{Results}

By 21 August (week 33), 37 laboratory-diagnosed cases of WNV infection were reported to the HCDCP; 31 of these (24 confirmed and seven probable) presented as WNND cases and six of them (all probable) as non-neuroinvasive. This report focuses mainly on the 31 WNND cases, which were identified and reported more consistently, because of the disease severity. The overall incidence of WNND in the country was 0.28 cases per 100,000 population (Table).

For the 37 laboratory confirmed cases, 31 serum samples and 25 CSF specimens were available; for 19 patients both CSF and serum specimens were provided, while for six patients only CSF was available. WNV-specific IgM antibodies were detected in all 31 serum samples and in 24 CSF specimens, while WNVspecific IgG antibodies were detected in 15 serum and eight CSF specimens. In all 19 patients for whom both types of specimen were available, WNV-specific IgM antibodies were detected in both CSF and serum. As was the case in 2010, all specimens were negative for TBEV, while low level of cross-reactivity was seen in
IgM with DENV [12]. None of the patients had been vaccinated for yellow fever.

The first case of WNND reported onset of symptoms on 16 July 2011 (week 28) (Figure 1). An increased number of cases was observed during weeks 30 and 32.

The median age of patients with WNND was 70 years (range 21-87) with the age-specific attack rate of WNND increasing significantly $(p=0.009)$ with increasing age. The incidence in persons aged 70 years or older was approximately 23 times higher compared to that of individuals younger than 30 years (Table). Of all WNND cases, 19 were male. The place of residence of WNND cases is presented in Figure 2.

None of the cases reported travel abroad during the incubation period. The first cases occurred in northern Greece in Central Macedonia and Thessalia region, whereas approximately 10 days later, cases were reported for the first time from Eastern Attiki (in close proximity - approximately $43 \mathrm{~km}$ - to the metropoli$\tan$ area of Athens). Overall, cases were distributed throughout nine of 54 Greek districts in four of 13 Greek regions. Of all WNND cases, 17 occurred in areas where cases had not been documented in 2010 (namely Karditsa and Trikala in Thessalia region, and Eastern Attiki and Viotia, in Central Greece). None of the cases had a history of recent blood transfusion or tissue/ organ transplantation.

\section{TABLE}

Basic characteristics of reported cases of West Nile neuroinvasive disease, Greece, 16 July - 21 August 2011 (n=31)

\begin{tabular}{|c|c|c|c|}
\hline Characteristic & Number of cases & $\begin{array}{l}\text { Incidence rate }{ }^{\mathrm{a}} \text { per } \\
100,000 \text { population) }\end{array}$ & $\begin{array}{c}\text { Risk Ratio } \\
\text { (95\% confidence interval) }\end{array}$ \\
\hline \multicolumn{4}{|l|}{ Age group (years) } \\
\hline$\ll 30$ & 2 & 0.05 & reference \\
\hline $30-49$ & 2 & 0.06 & $1.10(0.15-7.78)$ \\
\hline $50-59$ & 3 & 0.21 & $3.91(0.65-23.41)$ \\
\hline $60-69$ & 5 & 0.42 & $7.77(1.51-40.03)$ \\
\hline$\geq 70$ & 19 & 1.26 & $23.30(5.43-100.04)$ \\
\hline \multicolumn{4}{|l|}{ Sex } \\
\hline Female & 12 & 0.21 & reference \\
\hline Male & 19 & 0.34 & $1.61(0.78-3.33)$ \\
\hline \multicolumn{4}{|c|}{ District/prefecture (region) of residence } \\
\hline Karditsa (Thessalia) & 5 & 4.30 & $12.26(3.29-45.66)$ \\
\hline Eastern Attiki (Attiki) & 10 & 2.48 & $7.05(2.21-22.49)$ \\
\hline Serres (Central Macedonia) & 3 & 1.59 & $4.54(1.02-20.27)$ \\
\hline Larissa (Thessalia) & 4 & 1.40 & $3.99(1.00-15.95)$ \\
\hline Imathia (Central Macedonia) & 2 & 1.39 & $3.95(0.72-21.58)$ \\
\hline Viotia (Sterea Ellada) & 1 & 0.80 & $2.27(0.25-20.32)$ \\
\hline Trikala (Thessalia) & 1 & 0.77 & $2.18(0.24-19.50)$ \\
\hline Pella (Central Macedonia) & 1 & 0.69 & $1.96(0.22-17.57)$ \\
\hline Thessaloniki (Central Macedonia) & 4 & 0.35 & reference \\
\hline Total & 31 & 0.28 & \\
\hline
\end{tabular}

a Incidence rates were calculated using as denominator the 2007 mid-year population estimates of the respective groups available from the Hellenic Statistical Authority. 
Of all WNND cases, 22 presented with meningoencephalitis and nine with meningitis alone. Two patients presented with a combination of meningoencephalitis and acute flaccid paralysis. Information on underlying chronic medical conditions was available for 23 of the WNND cases: 17 had at least one underlying disease, with the most common being hypertension $(n=11)$, diabetes mellitus $(n=7)$ and coronary artery disease $(n=6)$. All WNND cases were hospitalised and six were admitted to an intensive care unit (ICU). As of 21 August 2011, one case (aged over 70 years) who had several underlying conditions, had a fatal outcome.

There were also six non-WNND cases reported, who probably represent a very small fraction of all nonWNND, as mild WNV cases are less likely to seek medical care and be identified. In depth interviews were conducted with all of them. The median age of the reported non-WNND cases was 44 years (range 10-78) and was significantly different $(p=0.009)$ from that of non-WNND ones (median age 70 years; range 21-87). Of those, five were hospitalised but none in an ICU. With regard to specific symptoms, fever was reported by all of them, followed by headache $(n=3)$, rash $(n=2)$, weakness $(n=2)$ and nausea/vomiting or diarrhoea $(n=1)$.

\section{FIGURE 1}

Reported cases of West Nile neuroinvasive disease by week of symptom onset, Greece, 16 July - 21 August 2011 ( $n=31)$

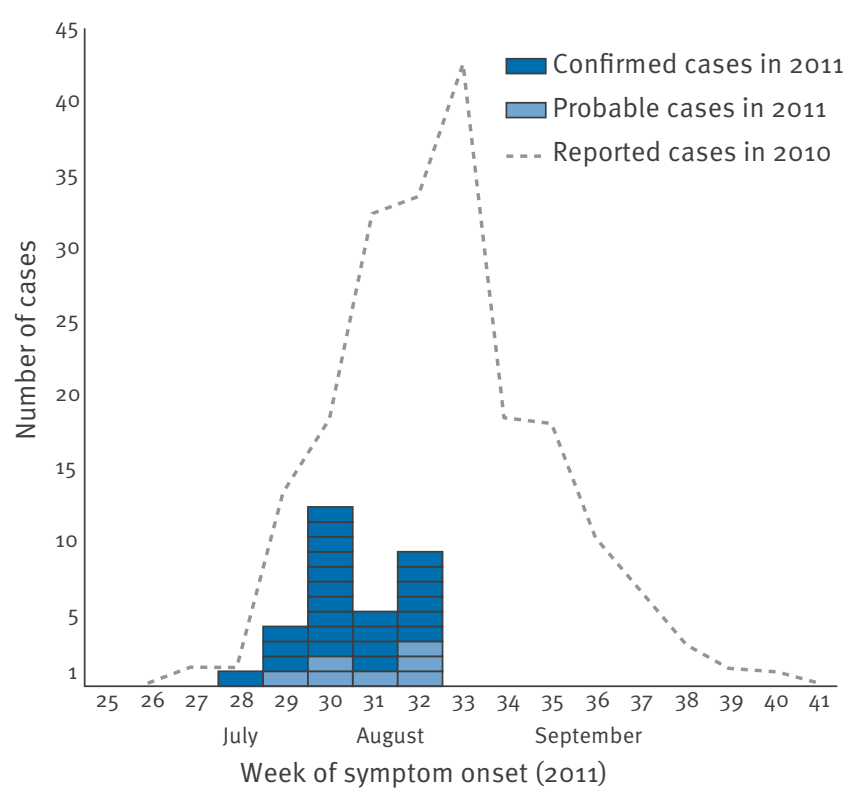

As this is an ongoing outbreak, the number of 2011 cases corresponding to previous weeks may increase as more cases are confirmed retrospectively.

Source: Hellenic Centre for Disease Control and Prevention.

\section{FIGURE 2}

Place of residence of reported cases of West Nile neuroinvasive disease, Greece, 16 July - 21 August 2011 (n=31)

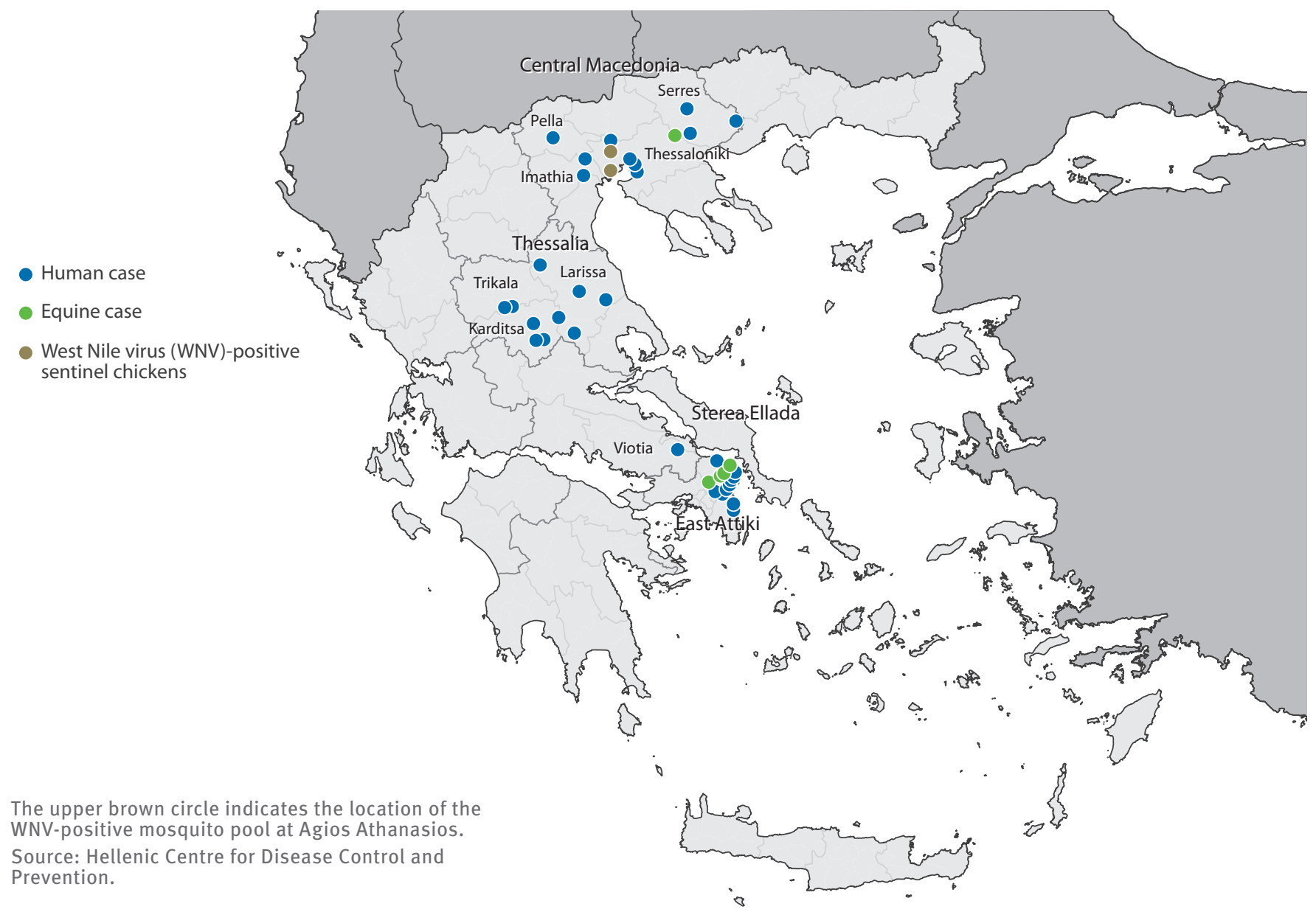




\section{Discussion and conclusions}

Following the large WNV outbreak in 2010, WNV human infections are notified in Greece for a second consecutive year. As of 21 August, 31 WNND were reported from four regions in Greece. Human cases of WNV infection were also detected in other European and Mediterranean countries during this season (as of 18 August, two cases had been reported in Romania, 21 in the Russian Federation, two in Albania, and five in Israel) [13].

The 2010 outbreak in Greece was mainly localised in Central Macedonia. Although cases seem to reoccur in the same districts as in 2010, a new geographic pattern of WNV spread is being observed in 2011. Following the intense WNV amplification and transmission in Central Macedonia in the previous year, the virus seems to disperse southward to the newly affected areas of Thessalia region (Karditsa) and further south to East Attiki (approximately $500 \mathrm{~km}$ from Central Macedonia), in proximity to the metropolitan area of Athens. Similar dispersal patterns have been observed in California, which has a similar climate to Greece, where WNV was introduced in 2003 and quickly spread throughout the state $[14,15]$.

The temporal distribution of cases shows that the first human cases in 2011 occurred approximately two weeks later compared to 2010. Comparing the magnitude of the current outbreak in Central Macedonia to the outbreak in the previous year, the current one remains lower to date, suggesting decreased or delayed WNV transmission in humans. Due to the high visibility of the 2010 outbreak and the subsequent raised awareness to the infection among physicians, it is unlikely to be due to delayed recognition of the disease or under-reporting. However, as this is an ongoing outbreak, the number of 2011 cases corresponding to previous weeks may increase as more cases are confirmed retrospectively.

In early May and June 2011, WNV transmission was detected by seroconversions of sentinel chickens and domestic pigeons in Central Macedonia [15]. Lineage 2 WNV sequences were obtained from one pool of Culex pipiens mosquitoes trapped in the city of Agios

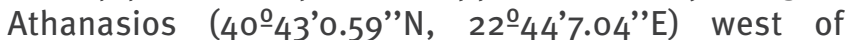
Thessaloniki on 23 June 2011. This strain showed the highest homology (99.4\%) to the Nea Santa/ Greece/2010 WNV strain detected in Culex pipiens in 2010. An identical strain was also detected in seroconverted sentinel chickens in the same city on 13 July 2011 [15]. Genetic characterisation of WNV strain(s) of 2011 circulating in other areas will elucidate whether it is an identical strain or a newly introduced one.

Regarding equidae, five horses from East Attiki presented with clinical WNV disease manifestation, as well as one horse from Serres (Central Macedonia) with antibodies against WNV that demonstrated a recent infection (IgM positive) (Figure 2) [16]. The first two cases in horses were identified before human cases had been reported in Attiki and thus functioned as an early warning signal.

Following the large 2010 WNV infection outbreak, a number of public health measures were implemented in 2011:

- guidelines for healthcare professionals for the recognition, management and diagnosis of encephalitis and WNV infection in order to improve their awareness regarding the disease;

- enhanced surveillance of encephalitis and WNV infection in humans;

- a project on mosquito mapping across the country;

- a project on mosquito surveillance;

- a seroprevalence study of WNV infection among humans in the epicentre of the 2010 WNV infection outbreak;

- a WNV seroepidemiological study in domestic pigeons and poultry;

- multi-sectoral collaboration and exchange of information between human health, veterinary health and entomological sectors;

- guidance for blood and blood product safety according to the EU directives;

- communication and health promotion activities encouraging personal protection against mosquito bites in the general population;

- vector control activities.

In conclusion, the reoccurrence of human WNV infection cases in two consecutive years and the spread of the virus in newly affected areas, suggest that WNV is established in Greece and transmission may continue in the future. Intensified vector mosquito control programmes, along with ongoing public health education, integrated human and animal WNV surveillance to monitor the spread of the virus and implementation of blood transfusion measures are necessary to prevent transmission and control the disease.

Updates on reported WNV cases in Greece are published in the Weekly Surveillance Reports available in English on the HCDCP website [17].

\section{Acknowledgments}

We would like to thank all hospital physicians and local public health authorities who contributed to the surveillance of WNV infections in Greece; the personnel of the University and Hospital laboratories for technical support; and Athanasiou M, Detsis M, Kallimani A, Sapounas S, Balaska A, Ntakou A, for their support in surveillance activities.

References

1. Danis K, Papa A, Theocharopoulos G, Dougas G, Athanasiou M, Detsis M, et al. West Nile virus infection in Greece, 2010. Emerg Infect Dis. Forthcoming 2011.

2. Papa A, Danis K, Baka A, Bakas A, Dougas G, Lytras T, et al. Ongoing outbreak of West Nile virus infections in humans in Greece, July - August 2010. Euro Surveill. 2010;15(34):pii=19644. Available from: http://www. eurosurveillance.org/ViewArticle.aspx?Articleld=19644 
3. Tsai TF, Popovici F, Cernescu C, Campbell GL, Nedelcu NI. West Nile encephalitis epidemic in southeastern Romania. Lancet. 1998;352(9130):767-71.

4. Papa A, Xanthopoulou K, Gewehr S, Mourelatos S. Detection of West Nile virus lineage 2 in mosquitoes during a human outbreak in Greece. Clin Microbiol Infect. 2011;17(8):1176-80.

5. Papa A, Bakonyi T, Xanthopoulou K, Vásquez A, Tenorio A, Nowotny N. Genetic characterization of a neuroinvasive lineage 2 West Nile virus, Greece, 2010. Emerg Infect Dis. 2011;17(5):920-2.

6. Valiakos G, Touloudi A, lacovakis C, Athanasiou L, Birtsas P, Spyrou V, et al. Molecular detection and phylogenetic analysis of West Nile virus lineage 2 in sedentary wild birds (Eurasian magpie), Greece, 2010. Euro Surveill. 2011;16(18):pii=19862. Available from: http://www.eurosurveillance.org/ViewArticle. aspx?Articleld $=19862$

7. Pavlatos M, Smith CE. Antibodies to arthropod-borne viruses in Greece. Trans R Soc Trop Med Hyg. 1964;58:422-4.

8. Antoniadis A, Alexiou-Daniel S, Malissiovas N, Doutsos J, Polyzoni T, LeDuc JW, et al. Seroepidemiological survey for antibodies to arboviruses in Greece. Arch Virol. 1990 [suppl 1]: 277-285.

9. Papa A, Perperidou P, Tzouli A, Castiletti C. West Nile virusneutralizing antibodies in humans in Greece. Vector Borne Zoonotic Dis. 2010;10(7):655-8.

10. European Commission. Commission Decision of 28 April 2008 amending Decision 2002/253/EC laying down case definitions for reporting communicable diseases to the Community network under Decision No 2119/98/EC of the European Parliament and of the Council. 18.06.2008:L 159. Available from: http://ec.europa.eu/health/ph_threats/com/ docs/1589_2008_en.pdf

11. Hellenic Statistical Authority (EL. STAT.). Mid-year estimated population by 5 -year age groups level NUTS III (NOMOS). Population by usual residence. Pireus: EL. STAT. [Accessed 25 Aug 2011]. Available from: http://www.statistics.gr/ portal/page/portal/ver-1/ESYE/BUCKET/A1602/Other/ A1602_SPO18_TS_AN_00_1991_00_2007_08_F_EN.pdf

12. Papa A, Karabaxoglou D, Kansouzidou A. Acute West Nile virus neuroinvasive infections: cross-reactivity with dengue virus and tick-borne encephalitis virus. J Med Virol. 2011;83(10):1861-5.

13. European Centre for Disease Prevention and Control (ECDC). West Nile fever maps. Reported cases of West Nile fever for the EU and neighbouring countries. Stockholm: ECDC. [Accessed 20 Aug 2011]. Available from: http://ecdc.europa.eu/en/ activities/diseaseprogrammes/emerging and vector borne diseases/Pages/West_Niles_fever_Risk_Maps.aspx

14. Reisen W, Lothrop H, Chiles R, Madon M, Cossen C, Woods $\mathrm{L}$, et al. West Nile virus in California. Emerg Infect Dis. 2004;10(8):1369-78.

15. Chaskopoulou A, Dovas Cl, Chaintoutis SC, Bouzalas I, Ara G, Papanastassopoulou M. Evidence of enzootic circulation of West Nile virus (Nea Santa-Greece-2010, lineage 2), Greece, May to July 2011. Euro Surveill. 2011;16(31):pii=19933. Available from: http://www.eurosurveillance.org/ViewArticle. aspx?Articleld $=19933$

16. World Organisation for Animal Health (OIE). West Nile Fever, Greece. Paris: OIE. [Accessed: 25 Aug 2011. Available from: http://web.oie.int/wahis/public. php?page $=$ single_report $\&$ pop $=1 \&$ reportid $=10914$

17. Hellenic Centre for Disease Control and Prevention (HCDCP). West Nile Virus. Weekly Epidemiological Report. Athens: HCDCP. [Accessed 25 Aug 2011]. Available from: http://www.keelpno.gr/index.php?option $=$ com content\&view $=$ article \&id $=365: 2011-07-11-11-53-54 \&$ catid $=72$ 